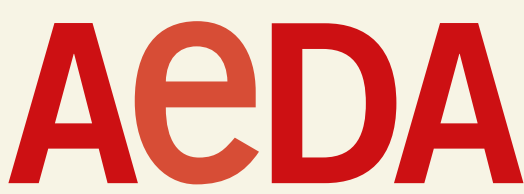

Ärzteverband Deutscher Allergologen (ÄDA)

Verantwortlich für den Inhalt

Prof. Dr. Ludger Klimek

Zentrum für Rhinologie und Allergologie An den Quellen 10, 65183 Wiesbaden

Tel.: (06 11) 8804799

Fax: (06 11) 308608255

E-Mail: ludger.klimek@allergiezentrum.org

\section{ÄDA-Geschäftsstelle}

Service Systems

Blumenstraße 14, 63303 Dreieich

Tel.: (0 61 03) 62273

Fax: (0 61 03) 697019

E-Mail: info@aeda.de

www.aeda.de

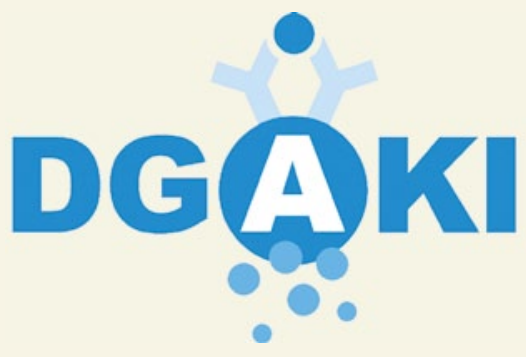

Deutsche Gesellschaft für Allergologie und klinische Immunologie (DGAKI)

\section{Verantwortlich für die Inhalte}

Prof. Dr. Thilo Jakob

Allergieabteilung und Forschergruppe Allergologie, Universitäts-Hautklinik Freiburg Hauptstraße 7, 79104 Freiburg

Tel.: (07 61) 2706714

Fax: (07 61) 2706655

E-Mail: thilo.jakob@uniklinik-freiburg.de

\section{DGAKI-Geschäftsstelle}

Gertrud Hammel

Schloss 2, 86482 Aystetten

Tel.: (08 21) 48687864

Fax: (08 21) 48687863

E-Mail: info@dgaki.de

www.dgaki.de

\title{
Leitfaden Hyposensibilisierungsbehandlung
}

\section{SIT verordnen ohne Regressrisiko}

\author{
Wer Ärzten wirklich Angst machen will, der drohe ihnen mit einer \\ Prüfung der Wirtschaftlichkeit ihrer Arzneimittelverordnungen. Damit \\ allergologisch tätige Mediziner, die eine spezifische Immuntherapie \\ verschreiben, nicht Gefahr laufen, bei einer solchen Prüfung in Regress \\ genommen zu werden, wurde jetzt ein Dokumentationsleitfaden \\ entwickelt.
}

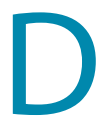
er Ärzteverband Deutscher Allergologen (ÄDA) hat Mitglieder in zahlreichen Arzneimittel-Regressverfahren beraten und begleitet. Dabei hat sich herausgestellt, dass auch bei Ärzten mit langjähriger allergologischer Erfahrung wesentliche Kriterien für die Indikationsstellung einer spezifischen Immuntherapie (SIT) nicht oder nicht in ausreichendem Maße bekannt waren.

\section{Voraussetzungen für SIT}

Die Durchführung einer SIT setzt stets voraus, dass es sich bei den zu behandelnden Erkrankungen um eine IgE-vermittelte Allergie vom Soforttyp handelt. Ergänzend ist bei der Indikationsstellung der Schweregrad der Symptomatik und die Effektivität der Therapie zu berücksichtigen (u. a. ARIA-Kritrien).

Gerade auf die korrekte Indikationsstellung und auf die Auswahl der eingesetzten Präparate richten die Prüfinstanzen ihr besonderes Augenmerk. Im Einzelfall sollte die Auswahl der Präparate immer auch Evidenzgrad und Wirkstoffgehalt (Studienlage!) berücksichtigen. Kostengünstig bedeutet nicht immer auch wirtschaftlich im Sinne des Sozialgesetzbuches V.

\section{Dokumentationsleitfaden entwickelt}

Bedauerlicherweise haben die bundesweit durchgeführten Prüfverfahren mit Regressandrohung viele Ärzte verunsichert. In der Folge werden Hyposensibilisierungen häufig gar nicht mehr durchgeführt.

Vor diesem Hintergrund hat ÄDAVorstand Prof. Dr. Wolfgang Wehrmann einen Dokumentationsleitfaden erarbeitet, der helfen soll, mehr Sicherheit in der Indikationsstellung für eine Hyposensibilisierungsbehandlung zu gewinnen. Er macht die Entscheidung für eine SIT leitliniengerecht nachvollziehbar und mindert so das Risiko, in einem Prüfungsverfahren in Regress genommen zu werden.

Ulrich Glatzer

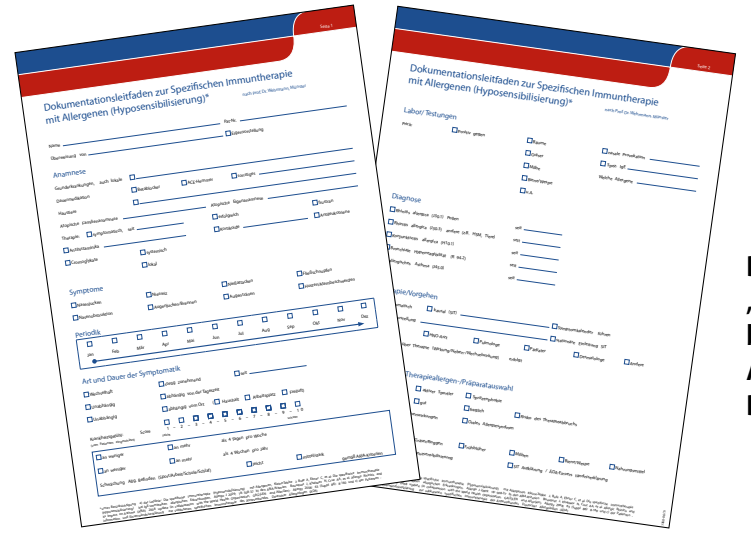

Der Leitfaden "Hyposensibilisierungsbehandlung" steht unter der Adresse www.aeda.de zum Download zur Verfügung. 